\title{
Parameterized fairness axioms on cycle-free graph games
}

\author{
S. Ryuo - K. Sato - Y. Yamamoto
}

Received: date / Accepted: date

\begin{abstract}
We study cooperative transferable utility games with a communication structure represented by an undirected graph, i.e., a group of players can cooperate only if they are connected on the graph. This type of games is called graph games and the best-known solution for them is the Myerson value, which is characterized by the component efficiency axiom and the fairness axiom. Recently the average tree solution has been proposed on cycle-free graph games, and shown to be characterized by the component efficiency axiom and the component fairness axiom. We propose $\epsilon$-parameterized fairness axiom on cyclefree graph games that incorporates the preceding fairness axioms, and show the existence and the uniqueness of the solution. We then discuss a relationship between the existing and our proposed solutions by a numerical example.
\end{abstract}

Keywords Cycle-free graph $\cdot \mathrm{TU}$-game $\cdot$ Communication structure $\cdot$ Fairness

\section{Introduction}

In many situations, a group of players obtains profits or saves costs by their cooperation. A subgroup of the players is called a coalition and the total profit yielded when all the members in the coalition agree to cooperate is called the worth of the coalition. The problem of how much payoff should be allocated to each player then arises if we know the worth of all possible coalitions. A classical set-valued solution is the core, being the set of payoffs at which the worth of the whole set of players is distributed among all the players and no coalition receives less than its worth. The best-known single-valued solution is the Shapley value [9], which is the average of all marginal contributions over all permutations of the player set. See, for example [3].

Satoko Ryuo $\cdot$ Keisuke Sato

Transport Information Technology Division, Railway Technical Research Institute, 2-8-38 Hikari-cho, Kokubunji-shi, Tokyo 185-8540, Japan

E-mail: ryuo@rtri.or.jp / keisato@rtri.or.jp

Yoshitsugu Yamamoto

Graduate School of Systems and Information Engineering, University of Tsukuba, Tsukuba, Ibaraki 3058537, Japan

E-mail: yamamoto@sk.tsukuba.ac.jp 
In this paper we consider this problem with restricted cooperation structure. Friendship could be an example of restricted cooperation. Complete strangers A and B might form a friendship in the presence of their mutual friend $\mathrm{C}$. We do not know the worth of the coalition consisting of A and B, while that of A, B and C is conceivable. This restricted cooperation structure is often represented by undirected graphs, and cooperative transferable utility games with such structure are called graph games. Myerson [7] introduced the Myerson value for the games and characterized it by component efficiency and fairness axioms. According to the result in Myerson [8], fairness can be replaced with balanced contribution in the context of the graph games. The position value proposed by Borm and Tijs [2] is another solution for the games. This value is characterized by component efficiency and balanced link contribution, which shares an idea with balanced contribution, see also Slikker [11]. Both the Myerson value and the position value are based on the Shapley value. In Slikker and van den Nouweland [10] the properties of these values are described in detail. Recently Herings et al. [5] proposed the average tree solution on the class of cycle-free graph games and characterized it by component efficiency and component fairness. They also showed that the solution is in the core if the game exhibits super-additivity, while the Myerson value or the position value may not. The average tree solution is the average of the tree solutions introduced by Demange [4] (in the paper it is called the hierarchical outcomes). She showed that the tree solutions yield extreme points of the core on a super-additive cycle-free graph game. The condition of super-additivity was relaxed to a weaker one by Talman and Yamamoto [12]. In Herings et al. [6] the average tree solution was generalized on the whole class of graph games, however no characterization was given there.

Fairness axiom means that, when a link is deleted from the underlying graph, the two players on the ends of the link will get the same loss or gain in payoff. Meanwhile, component fairness requires that the average loss or gain per player in one of the detached components is equal to that in the other. We introduce $\epsilon$-parameterized fairness axiom that incorporates these axioms of fairness, and show the existence and the uniqueness of a solution satisfying component efficiency and our axiom. We then discuss a relationship among the existing solutions, our proposed solutions and the core by a numerical example.

The rest of the paper is organized as follows. Sect. 2 lays out graph games. In Sect. 3 we review the Myerson value and the average tree solution along with the axioms characterizing them, and the relationship between the solutions and the core. We propose a new fairness axiom in Sect. 4 that incorporates the preceding two axioms on cycle-free graph games and discuss the existence and the uniqueness of a solution satisfying our axiom. In Sect. 5, we give an example of graph games of three players, and compare the existing solutions, our proposed solutions and the core.

\section{Preliminaries}

A cooperative game with transferable utility or simply a $T U$-game is defined by a pair $(N, v)$, where $N$ is a finite set of players, i.e., $N=\{1,2, \ldots, n\}$, and $v: 2^{N} \rightarrow \mathbb{R}$ is a characteristic function such that $v(\varnothing)=0$. The worth of a coalition $S \in 2^{N}$ is denoted by $v(S)$. We denote the game $(N, v)$ by $v$ for short and the collection of all characteristic functions by $\mathcal{V}$. A payoff vector $\boldsymbol{x} \in \mathbb{R}^{n}$ is an $n$-dimensional real vector, and we let $x(S):=\sum_{i \in S} x_{i}$ for each $S \in 2^{N}$, where $x_{i}$, the $i$ th component of $\boldsymbol{x}$, is player $i$ 's payoff. The best-known Shapley value [9] is represented by

$$
\psi(v):=\frac{1}{n !} \sum_{\pi \in \Pi} \boldsymbol{m}^{\pi}(v),
$$


where $\boldsymbol{m}^{\pi}(v) \in \mathbb{R}^{n}$ is called the marginal vector corresponding to a permutation $\pi$ and $\Pi$ is the set of all permutations on $N$. The $i$ th component $m_{i}^{\pi}(v)$ of $\boldsymbol{m}^{\pi}(v)$ is defined as $m_{i}^{\pi}(v):=$ $v\left(\pi^{i} \cup\{i\}\right)-v\left(\pi^{i}\right)$, where $\pi^{i}:=\{j \in N \mid \pi(j)<\pi(i)\}$, i.e., the set of players preceding $i$ in permutation $\pi$.

Next we give several notations for an undirected graph $(N, L)$ with node set $N$ and edge set $L$. The collection of sets of edges is denoted by $\mathcal{L}$. For $K \in 2^{N}$, the graph $(K, L(K))$, where $L(K):=\{\{i, j\} \mid i, j \in K,\{i, j\} \in L\}$, is called the subgraph of $(N, L)$ on $K$. Two nodes $i, j \in N$ are connected in $(N, L)$ if either $i=j$ or there exists a sequence of edges $\left\{i_{0}, i_{1}\right\},\left\{i_{1}, i_{2}\right\}, \ldots,\left\{i_{l-1}, i_{l}\right\}$ such that $i_{0}=i$ and $i_{l}=j$. A graph $(N, L)$ or simply $N$ is connected if any two nodes $i, j \in N$ are connected in $(N, L)$. A subset $K \subseteq N$ is said to be a connected subset of $N$ when the subgraph $(K, L(K))$ is connected. The collection of all connected subsets of $K$ in $(K, L(K))$ is denoted by $C(K, L)$, i.e., $C(K, L):=\{H \mid H \subseteq$ $K$ and $H$ is a connected subset of $K\}$. A subset $K$ of $N$ is a component of $(N, L)$ if $K$ is maximally connected, i.e., $K$ is connected but $K \cup\{j\}$ is not for any $j \in N \backslash K$. The collection of all components of $(K, L(K))$ is denoted by $C_{m}(K, L)$, i.e., $C_{m}(K, L):=\{H \mid H \subseteq$ $K$ and $H$ is a component of $K\}$. A sequence of edges $\left\{i_{0}, i_{1}\right\},\left\{i_{1}, i_{2}\right\}, \ldots,\left\{i_{l}, i_{l+1}\right\}$ is a cycle in $(N, L)$ if

(i) $l \geq 2$,

(ii) $i_{l+1}=i_{0}$, and

(iii) $\left\{i_{h}, i_{h+1}\right\} \in L$ for $h=0, \ldots, l$.

A graph $(N, L)$ is cycle-free if it does not contain any cycle. Here we define the collection of sets of edges forming a cycle-free graph as $\mathcal{M}$.

A graph game is given by a triple $(N, v, L)$ where $N$ is a set of players, $v$ is a characteristic function and $L$ is a set of edges of the graph $(N, L)$. Omitting $N$, we denote the graph game simply by $(v, L)$, the collection of all graph games by $\mathcal{V} \times \mathcal{L}$ and that of cycle-free graph games by $\mathcal{V} \times \mathcal{M}$. On graph games only connected subsets of the players are able to cooperate, hence the set of admissible coalitions is $C(N, L)$. When the graph $(N, L)$ is complete, i.e., any two of its nodes are connected by an edge, graph game turns out to be an ordinary TU-game. In this paper we assume that $N$ is connected in $(N, L)$, i.e., $N \in C(N, L)^{1}$. A function $f: \mathcal{V} \times \mathcal{L} \rightarrow \mathbb{R}^{n}$ is called a solution for graph games and $f_{i}(v, L)$ is called player $i$ 's allocation by solution $f$. The core of the graph game is given by

$$
C(v, L):=\left\{\boldsymbol{x} \in \mathbb{R}^{n} \mid x(N)=v(N) \text { and } x(S) \geq v(S) \text { for all } S \in C(N, L)\right\}
$$

The restricted game $v^{L}$ is defined by Myerson [7] as

$$
v^{L}(S):=\sum_{T \in C_{m}(S, L)} v(T) \quad \text { for each } S \in 2^{N} .
$$

\section{Existing Solutions}

We introduce the Myerson value and the average tree solution, and then the axioms which characterize each of them. In the followings we use the abbreviation $L \backslash\{i, j\}$ to denote the set difference of $L$ and $\{\{i, j\}\}$.

\footnotetext{
1 Otherwise, we have only to discuss the problem on each component separately.
} 
Definition 1 On the class of all graph games, the Myerson value, denoted by $\mu(v, L)$, is the Shapley value of the restricted game defined by (3), i.e.,

$$
\mu(v, L):=\psi\left(v^{L}\right) .
$$

As will be stated in Theorem 1 below, the Myerson value is characterized by the following two axioms.

Axiom 1 (component efficiency) For any $(v, L) \in \mathcal{V} \times \mathcal{L}$ it holds that

$$
\sum_{i \in N} f_{i}(v, L)=v(N)
$$

Axiom 2 (fairness) For any $(v, L) \in \mathcal{V} \times \mathcal{L}$ and $\{i, j\} \in L$, it holds that

$$
f_{i}(v, L)-f_{i}(v, L \backslash\{i, j\})=f_{j}(v, L)-f_{j}(v, L \backslash\{i, j\}) .
$$

Component efficiency means that the sum of the players' allocations in a component is equal to the worth of the component. Note that this axiom is assumed to be satisfied by all the solutions that we will introduce in this paper. Fairness means that the two players connected by an edge obtain the same change of allocation if the edge is deleted. Myerson gave the following theorem.

Theorem 1 (Myerson [7]) On the class of all graph games, the Myerson value $\mu$ is the unique solution that satisfies Axiom 1 and Axiom 2.

The average tree solution is a solution which was first introduced by Herings et al. [5] for cycle-free graph games. To describe the solution we give here some definitions on a directed graph $(N, D)$ following Berge [1], where $D$ is a set of ordered pairs of nodes called $\operatorname{arcs}$. If $(i, j) \in D$, then we say that node $j$ is a successor of $i$ and we denote by $S_{D}^{\prime}(i)$ the set of $i$ 's successors. If $(j, i) \in D$, then we say that node $j$ is a predecessor of $i$ and we denote by $P_{D}^{\prime}(i)$ the set of $i$ 's predecessors. A sequence of $\operatorname{arcs}\left(i_{0}, i_{1}\right),\left(i_{1} . i_{2}\right), \ldots,\left(i_{l-1}, i_{l}\right)$ is a directed path from $i_{0}$ to $i_{l}$ in $(N, D)$ if $\left(i_{h}, i_{h+1}\right) \in D$ for $h=0, \ldots l-1$, while it is called a cycle if $\left(i_{h}, i_{h+1}\right) \in D$ for all $h$ and $i_{0}=i_{l}$. A graph $(N, D)$ is cycle-free if it does not contain any cycle. In the cycle-free graph, we say that $j$ is a subordinate of $i$ when there is a directed path from $i$ to $j$. The set of subordinates of $i$ is defined by $S_{D}(i)$ and we let $\bar{S}_{D}(i):=S_{D}(i) \cup\{i\}$. A node $r$ is called a root if all the nodes of $N \backslash\{r\}$ can be reached by directed paths starting from $r$ and it is not a successor of any other node. A leaf is a node having no successor. Finally we say that $D^{r}$ is an arborescence or a rooted tree with respect to node $r \in N$ when $r$ is a root and the other nodes have only one predecessor, i.e., $\left|P_{D^{r}}^{\prime}(i)\right|=1$ for all $i \in N \backslash\{r\}$ on the graph $\left(N, D^{r}\right)$.

Given a cycle-free graph game $(v, L)$, we define a tree solution with respect to $r \in N$ as follows and denote it by $\boldsymbol{x}^{r}$ : make an arborescence $D^{r}$ with node $r$ as the root by giving a direction to all the edges in $L$. Note that $D^{r}$ is uniquely determined for each $r \in N$ since the original undirected graph $(N, L)$ is cycle-free and connected. For each node $i \in N$ the $i$ th component of the tree solution $\boldsymbol{x}^{r}$ is given by

$$
x_{i}^{r}:=v^{L}\left(\bar{S}_{D^{r}}(i)\right)-v^{L}\left(S_{D^{r}}(i)\right) .
$$

There are $n$ different tree solutions thus obtained and the average tree solution is defined as follows. 
Definition 2 On the class of cycle-free graph games, the average tree solution $A T(v, L)$ is the average of all tree solutions, i.e.,

$$
A T(v, L):=\frac{1}{n} \sum_{r \in N} \boldsymbol{x}^{r}
$$

Herings et al. [5] replaced the fairness axiom by the component fairness axiom below to provide a characterization of the average tree solution. Let $f: \mathcal{V} \times \mathcal{M} \rightarrow \mathbb{R}^{n}$ be a solution for cycle-free graph games. For a cycle-free undirected graph $(N, L)$ and an edge $\{i, j\} \in L$ let $K^{i}$ and $K^{j}$ be the components of $(N, L \backslash\{i, j\})$ containing node $i$ and node $j$, respectively.

Axiom 3 (component fairness) For any $(v, L) \in \mathcal{V} \times \mathcal{M}$ and $\{i, j\} \in L$, it holds that

$$
\frac{1}{\left|K^{i}\right|} \sum_{h \in K^{i}}\left(f_{h}(v, L)-f_{h}(v, L \backslash\{i, j\})\right)=\frac{1}{\left|K^{j}\right|} \sum_{h \in K^{j}}\left(f_{h}(v, L)-f_{h}(v, L \backslash\{i, j\})\right) .
$$

Component fairness means that deleting the edge between two nodes yields the same average loss in payoff between the divided two components.

Theorem 2 (Herings et al. [5]) On the class of cycle-free graph games, the average tree solution AT is the unique solution that satisfies Axiom 1 and Axiom 3.

Herings et al. [5] also discuss the relationship between the average tree solution and the core of graph games. A graph game is said to be super-additive if

$$
v(S \cup T) \geq v(S)+v(T)
$$

holds for all $S, T \in C(N, L)$ such that $S \cap T=\varnothing$ and $S \cup T \in C(N, L)$. It is shown in Demange [4] that tree solution $\boldsymbol{x}^{r}$ is one of the extreme points of the core for any $r$ on superadditive cycle-free graph games. Hence the average tree solution is in the core of the graph game, i.e., $A T(v, L) \in C(v, L)$, while the Myerson value is not always in it.

\section{Parameterized Fairness Axiom and Existence of Solution}

This section presents a new axiom of fairness for cycle-free graph games that incorporates the preceding ones, and shows the existence and the uniqueness of the solution satisfying component efficiency and our fairness axiom. We focus on the difference between Axiom 2 and Axiom 3 in the coefficients of $f_{h}(v, L)$ for $h \in N$. Fairness requires the loss of player $i$ be equal to that of player $j$ when it is caused by the deletion of edge $\{i, j\}$. On the other hand, component fairness requires the average loss per player in one component be equal to that in the other component. It seems, however, unreasonable that a player away from the deleted edge is assigned the same loss coefficient as a player close to the edge. Therefore we propose a new fairness axiom under which the coefficient varies according to the distance from the deleted edge by introducing a nonnegative parameter $\epsilon$. We name it $\epsilon$-parameterized fairness. 
Axiom 4 ( $\epsilon$-parameterized fairness) For any $(v, L) \in \mathcal{V} \times \mathcal{M}, \epsilon \geq 0$ and $\{i, j\} \in L$, it holds that

$$
\begin{aligned}
\frac{1}{\sum_{h \in K^{i}} \epsilon^{\tau^{i}(h)}} \sum_{h \in K^{i}} \epsilon^{\tau^{i}(h)}\left(f_{h}(v, L)-f_{h}(v, L \backslash\{i, j\})\right) \\
=\frac{1}{\sum_{h \in K^{j}} \epsilon^{\tau^{j}(h)}} \sum_{h \in K^{j}} \epsilon^{\tau^{j}(h)}\left(f_{h}(v, L)-f_{h}(v, L \backslash\{i, j\})\right),
\end{aligned}
$$

where $\tau^{i}(h)$ is defined as follows: for component $K^{i}$ make an arborescence $D^{i}$ with node $i$ as the root and set

$$
\tau^{i}(h):= \begin{cases}0 & \text { if } P_{D^{i}}^{\prime}(h)=\varnothing, \\ 1+\tau^{i}(k) \text { with }\{k\}=P_{D^{i}}^{\prime}(h) & \text { otherwise. }\end{cases}
$$

The function $\tau^{i}(h)$ is the depth of node $h$ in the arborescence $D^{i}$ with $i$ as the root, and the loss coefficient of a node is $\epsilon$ times that of his predecessor. Hence for $\epsilon<1$ the coefficient of a player close to the deleted edge is relatively high, while that of a player away from the deleted edge is low. The coefficients are normalized so that the sum of the coefficients in the same component is equal to one. If we set $\epsilon$ either to 0 or to 1 we obtain the preceding axioms.

Corollary 1 In Axiom 4, if we set $\epsilon=0$ then we obtain Axiom $2^{2}$ and if we set $\epsilon=1$ then we obtain Axiom 3.

The following theorem shows the existence and the uniqueness of the solution satisfying component efficiency and $\epsilon$-parameterized fairness.

Theorem 3 On the class of cycle-free graph games, for any $\epsilon \geq 0$ there exists a unique solution that satisfies Axiom 1 and Axiom 4.

Given a cycle-free graph $(N, L)$, we create, from the $n-1$ equations in Axiom 4 and the equation in Axiom 1, the following linear equality system

$A f(v, L)=\boldsymbol{b}$,

where the matrix $A \in \mathbb{R}^{n \times n}$ consists of the coefficients of $f_{h}(v, L), h \in N$, and the right hand side vector $\boldsymbol{b} \in \mathbb{R}^{n}$ consists of $v(N)$ and the terms of $f_{h}(v, L \backslash\{i, j\})$ for $\{i, j\} \in L$ and $h \in N$. For example, for the graph of $N=\{1,2,3\}$ and $L=\{\{1,2\},\{2,3\}\}$ we have the following three equations :

$$
\begin{aligned}
f_{1}(v, L)-f_{1}(v, L \backslash\{1,2\})= & \frac{1}{1+\epsilon}\left(f_{2}(v, L)-f_{2}(v, L \backslash\{1,2\})\right) \\
& +\frac{\epsilon}{1+\epsilon}\left(f_{3}(v, L)-f_{3}(v, L \backslash\{1,2\})\right), \\
f_{3}(v, L)-f_{3}(v, L \backslash\{2,3\})= & \frac{\epsilon}{1+\epsilon}\left(f_{1}(v, L)-f_{1}(v, L \backslash\{2,3\})\right) \\
+ & \frac{1}{1+\epsilon}\left(f_{2}(v, L)-f_{2}(v, L \backslash\{2,3\})\right), \\
f_{1}(v, L)+f_{2}(v, L)+f_{3}(v, L)=v(N) . &
\end{aligned}
$$

\footnotetext{
${ }^{2}$ We regard $0^{0}$ as 1 .
} 
By transposition of terms, we have

$$
\begin{aligned}
& f_{1}(v, L)-\frac{1}{1+\epsilon} f_{2}(v, L)-\frac{\epsilon}{1+\epsilon} f_{3}(v, L) \\
& \quad=f_{1}(v, L \backslash\{1,2\})-\frac{1}{1+\epsilon} f_{2}(v, L \backslash\{1,2\})-\frac{\epsilon}{1+\epsilon} f_{3}(v, L \backslash\{1,2\}), \\
& \frac{\epsilon}{1+\epsilon} f_{1}(v, L)+\frac{1}{1+\epsilon} f_{2}(v, L)-f_{3}(v, L) \\
& =\frac{\epsilon}{1+\epsilon} f_{1}(v, L \backslash\{2,3\})+\frac{1}{1+\epsilon} f_{2}(v, L \backslash\{2,3\})-f_{3}(v, L \backslash\{2,3\}), \\
& f_{1}(v, L)+f_{2}(v, L)+f_{3}(v, L)=v(N) .
\end{aligned}
$$

Thus the matrix $A$ reduces to

$$
\left[\begin{array}{ccc}
1 & -\frac{1}{1+\epsilon} & -\frac{\epsilon}{1+\epsilon} \\
\frac{\epsilon}{1+\epsilon} & \frac{1}{1+\epsilon} & -1 \\
1 & 1 & 1
\end{array}\right]
$$

each column corresponding to a node and each row corresponding to an edge to be deleted in Axiom 4 except for the last row that comes from the component efficiency. For inductive proof we assume that $f_{h}(v, L \backslash\{i, j\})$ exists and accordingly $\boldsymbol{b}$ is a constant vector ${ }^{3}$. We show that the system has a unique solution by proving that

$$
A c=\mathbf{0}
$$

holds only if $\boldsymbol{c}=\mathbf{0}$.

Let us choose an arbitrary node as the root and make an arborescence $D$ as we did in defining the tree solution in Sect. 3. Then we obtain the following two lemmas.

Lemma 1 Let $j_{1}$ and $j_{2}$ be successors of $i$, i.e., $\{i\}=P_{D}^{\prime}\left(j_{1}\right)=P_{D}^{\prime}\left(j_{2}\right)$. If $c_{p}=c_{j_{1}}$ for all $p \in \bar{S}_{D}\left(j_{1}\right)$ and $c_{q}=c_{j_{2}}$ for all $q \in \bar{S}_{D}\left(j_{2}\right)$, then $c_{j_{1}}=c_{j_{2}}$.

Proof Let $H=N \backslash\left(\bar{S}_{D}\left(j_{1}\right) \cup \bar{S}_{D}\left(j_{2}\right)\right)$. The corresponding equation for row $\left\{j_{1}, i\right\}$ in $A \boldsymbol{c}=\mathbf{0}$ is

$$
1 \times c_{j_{1}}-\frac{\sum_{h \in H} \epsilon^{\tau^{i}(h)} c_{h}+\sum_{q \in \bar{S}_{D}\left(j_{2}\right)} \epsilon^{\tau^{i}(q)} c_{j_{2}}}{\sum_{h \in H} \epsilon^{\tau^{i}(h)}+\sum_{q \in \bar{S}_{D}\left(j_{2}\right)} \epsilon^{\tau^{i}(q)}}=0,
$$

from $K^{j_{1}}=\bar{S}_{D}\left(j_{1}\right), K^{i}=H \cup \bar{S}_{D}\left(j_{2}\right)$ and our assumption. This is equivalent to

$$
\left(\sum_{h \in H} \epsilon^{\tau^{i}(h)}+\sum_{q \in \bar{S}_{D}\left(j_{2}\right)} \epsilon^{\tau^{i}(q)}\right) c_{j_{1}}-\sum_{h \in H} \epsilon^{\tau^{i}(h)} c_{h}-\sum_{q \in \bar{S}_{D}\left(j_{2}\right)} \epsilon^{\tau^{i}(q)} c_{j_{2}}=0 .
$$

For row $\left\{j_{2}, i\right\}$ we analogously have

$$
\left(\sum_{h \in H} \epsilon^{\tau^{i}(h)}+\sum_{p \in \bar{S}_{D}\left(j_{1}\right)} \epsilon^{\tau^{i}(p)}\right) c_{j_{2}}-\sum_{h \in H} \epsilon^{\tau^{i}(h)} c_{h}-\sum_{p \in \bar{S}_{D}\left(j_{1}\right)} \epsilon^{\tau^{i}(p)} c_{j_{1}}=0 .
$$

\footnotetext{
${ }^{3}$ For $f_{h}(v, \tilde{L})$ with $|\tilde{L}|=1$ it clearly holds that $f_{h}(v, \varnothing)=v(h)$.
} 
By subtracting (7) from (6) we obtain

$$
\begin{aligned}
& \left(\sum_{h \in H} \epsilon^{\tau^{i}(h)}+\sum_{q \in \bar{S}_{D}\left(j_{2}\right)} \epsilon^{\tau^{i}(q)}+\sum_{p \in \bar{S}_{D}\left(j_{1}\right)} \epsilon^{\tau^{i}(p)}\right) c_{j_{1}} \\
& \quad-\left(\sum_{h \in H} \epsilon^{\tau^{i}(h)}+\sum_{p \in \bar{S}_{D}\left(j_{1}\right)} \epsilon^{\tau^{i}(p)}+\sum_{q \in \bar{S}_{D}\left(j_{2}\right)} \epsilon^{\tau^{i}(q)}\right) c_{j_{2}}=0
\end{aligned}
$$

and see that $c_{j_{1}}=c_{j_{2}}$.

Lemma 2 Let $i$ be a node which has a successor $j$, i.e., $j \in S_{D}^{\prime}(i)$. If $c_{j}=c_{k}$ for all $k \in S_{D}(i)$, then $c_{i}=c_{j}$.

Proof When $i$ is the root, the values of the nodes other than $i$ are all $c_{j}$ by the assumption. The equation for row $\{j, i\}$ is then

$$
1 \times c_{j}-\frac{1}{\epsilon^{0}+\sum_{h \in K^{i} \backslash\{i\}} \epsilon^{\tau^{i}(h)}}\left(\epsilon^{0} c_{i}+\sum_{h \in K^{i} \backslash\{i\}} \epsilon^{\tau^{i}(h)} c_{j}\right)=0
$$

and it immediately holds that $c_{i}=c_{j}$.

When $i$ is not the root node, let $G=N \backslash \bar{S}_{D}(i)$ and the equation for row $\{j, i\}$ is, from $K^{j}=\bar{S}_{D}(j)$ and $K^{i}=\{i\} \cup\left(S_{D}(i) \backslash \bar{S}_{D}(j)\right) \cup G$,

$$
1 \times c_{j}-\frac{\epsilon^{0} c_{i}+\sum_{h \in S_{D}(i) \backslash \bar{S}_{D}(j)} \epsilon^{\tau^{i}(h)} c_{j}+\sum_{g \in G} \epsilon^{\tau^{i}(g)} c_{g}}{\epsilon^{0}+\sum_{h \in S_{D}(i) \backslash \bar{S}_{D}(j)} \epsilon^{\tau^{i}(h)}+\sum_{g \in G} \epsilon^{\tau^{i}(g)}}=0,
$$

which is equivalent to

$$
\left(\epsilon^{0}+\sum_{g \in G} \epsilon^{\tau^{i}(g)}\right) c_{j}-\epsilon^{0} c_{i}-\sum_{g \in G} \epsilon^{\tau^{i}(g)} c_{g}=0
$$

Next let $m$ be the predecessor of $i$ and consider the equation for row $\{i, m\}$. Since $K^{i}=$ $\{i\} \cup S_{D}(i)$ and $K^{m}=G$ in this case, we see that

$$
\frac{\epsilon^{0} c_{i}+\sum_{h \in S_{D}(i)} \epsilon^{\tau^{i}(h)} c_{j}}{\epsilon^{0}+\sum_{h \in S_{D}(i)} \epsilon^{\tau^{i}(h)}}-\frac{\sum_{g \in G} \epsilon^{\tau^{m}(g)} c_{g}}{\sum_{g \in G} \epsilon^{\tau^{m}(g)}}=0 .
$$

Since $\epsilon^{\tau^{m}(g)}=\epsilon^{\tau^{i}(g)-1}$ for $g \in G$, it is equivalent to

$$
\left(\sum_{g \in G} \epsilon^{\tau^{i}(g)-1}\right)\left(\epsilon^{0} c_{i}+\sum_{h \in S_{D}(i)} \epsilon^{\tau^{i}(h)} c_{j}\right)-\left(\epsilon^{0}+\sum_{h \in S_{D}(i)} \epsilon^{\tau^{i}(h)}\right)\left(\sum_{g \in G} \epsilon^{\tau^{i}(g)-1} c_{g}\right)=0 .
$$


By row operation $\left(\epsilon^{0}+\sum_{h \in S_{D}(i)} \epsilon^{\tau^{i}(h)}\right) \times(8)-\epsilon \times(9)$, we have

$$
\begin{array}{r}
\left(\epsilon^{0}+\sum_{h \in S_{D}(i)} \epsilon^{\tau^{i}(h)}\right)\left(\left(\epsilon^{0}+\sum_{g \in G} \epsilon^{\tau^{i}(g)}\right) c_{j}-\epsilon^{0} c_{i}\right)-\left(\sum_{g \in G} \epsilon^{\tau^{i}(g)}\right)\left(\epsilon^{0} c_{i}+\sum_{h \in S_{D}(i)} \epsilon^{\tau^{i}(h)} c_{j}\right) \\
=\left(\epsilon^{0}+\sum_{h \in S_{D}(i)} \epsilon^{\tau^{i}(h)}+\sum_{g \in G} \epsilon^{\tau^{i}(g)}\right) c_{j}-\left(\epsilon^{0}+\sum_{h \in S_{D}(i)} \epsilon^{\tau^{i}(h)}+\sum_{g \in G} \epsilon^{\tau^{i}(g)}\right) c_{i}=0
\end{array}
$$

and hence $c_{i}=c_{j}$.

Proof (Proof of Theorem 3) By applying Lemma 1 and Lemma 2 alternately from the leaves to the root of the arborescence, we see that $c_{i}=c_{j}$ for all $i, j \in N$. Since the system $A \boldsymbol{c}=\mathbf{0}$ includes the equation $\sum_{i \in N} c_{i}=0$ corresponding to Axiom 1, we have $\boldsymbol{c}=\mathbf{0}$ and conclude the proof.

The whole set of $\epsilon$-parameterized solutions incorporates the Myerson value and the average tree solution by Corollary 1 . The Myerson value is the centroid of the marginal vectors in the restricted games, and the average tree solution is the centroid of the tree solutions. However we do not know whether the $\epsilon$-parameterized solution is the centroid or the like of any known solution vectors, and we cannot provide a closed form of the $\epsilon$-parameterized solution.

\section{Example}

We will give an example of graph games and compare the three different solutions. The graph consists of $N=\{1,2,3\}$ and $L=\{\{1,2\},\{2,3\}\}$ as shown in Fig. 1 . The worths of connected sets are given by

$$
v(\{1\})=v(\{2\})=v(\{3\})=0, \quad v(\{1,2\})=0.8, \quad v(\{2,3\})=0.9 \quad \text { and } \quad v(\{1,2,3\})=1 .
$$

Note that this example satisfies super-additivity. The core of this game is given by

$$
x_{1}, x_{2}, x_{3} \geq 0, \quad x_{1}+x_{2} \geq 0.8, \quad x_{2}+x_{3} \geq 0.9 \quad \text { and } \quad x_{1}+x_{2}+x_{3}=1 .
$$

The tree solutions $\boldsymbol{x}^{r}$ for $r=1,2$ and 3, the Myerson value $\mu$ and the average tree solution $A T$ are displayed in Table 1. We also plot these solutions on the triangular graph in Fig. 2.

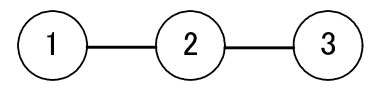

Fig. $1 \operatorname{Graph}(N, L)=(\{1,2,3\},\{\{1,2\},\{2,3\}\})$.

Table 1 Tree solutions, Myerson value and average tree solution.

\begin{tabular}{c|c|c|c|c|c|} 
& \multicolumn{4}{|c|}{ tree solution } & \\
& $\boldsymbol{x}^{1}$ & $\boldsymbol{x}^{2}$ & $\boldsymbol{x}^{3}$ & $\mu$ & $A T$ \\
\hline node 1 & 0.100 & 0.000 & 0.000 & 0.167 & 0.033 \\
node 2 & 0.900 & 1.000 & 0.800 & 0.617 & 0.900 \\
node 3 & 0.000 & 0.000 & 0.200 & 0.217 & 0.067
\end{tabular}




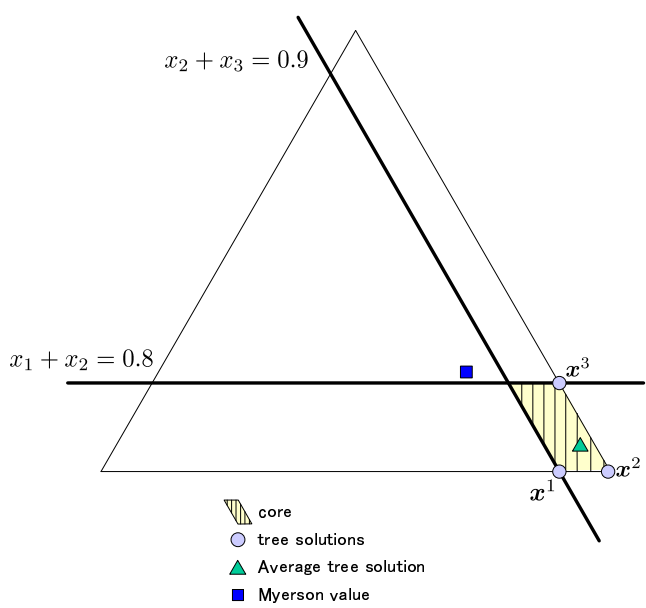

Fig. 2 Core and existing solutions.

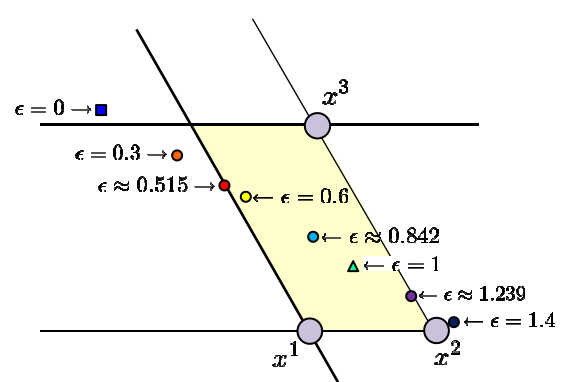

Fig. $3 \epsilon$-parameterized solutions.

Table $2 \epsilon$-parameterized solutions.

\begin{tabular}{c|c|c|c|c|c|c|c|c|}
$\epsilon$ & 0.0 & 0.3 & 0.515 & 0.6 & 0.842 & 1.0 & 1.239 & \multicolumn{1}{|c}{1.4} \\
\hline node 1 & 0.167 & 0.129 & 0.100 & 0.088 & 0.055 & 0.033 & 0.000 & -0.022 \\
node 2 & 0.617 & 0.702 & 0.763 & 0.787 & 0.855 & 0.900 & 0.968 & 1.013 \\
node 3 & 0.217 & 0.169 & 0.137 & 0.125 & 0.009 & 0.067 & 0.032 & 0.009
\end{tabular}

We observe that the tree solutions are extreme points of the core and the average tree solution is in it, while the Myerson value is out of it.

Fig. 3 and Table 2 show the $\epsilon$-parameterized solutions for several values of $\epsilon$. They form a continuous path connecting the Myerson value and the average tree solution, each of which corresponds to $\epsilon=0$ and $\epsilon=1$, respectively. In fact the $\epsilon$-parameterized solution is obtained explicitly as

$$
x_{1}=\frac{-17 \epsilon^{2}+13 \epsilon+10}{60(1+2 \epsilon)}, \quad x_{2}=\frac{17 \epsilon+37}{60}, \quad x_{3}=\frac{-17 \epsilon^{2}+16 \epsilon+13}{60(1+2 \epsilon)} .
$$

The payoff vector is in the core as long as $\epsilon$ remains in the interval between $(1+\sqrt{237}) / 34 \approx$ 0.515 and $(13+\sqrt{849}) / 34 \approx 1.239$. The average tree solution is in the convex hull of the tree solutions by its nature. In contrast the $\epsilon$-parameterized solutions can be out of this convex hull but lie in the left half of the core when $(1+\sqrt{237}) / 34 \leq \epsilon \leq(3+8 \sqrt{2}) / 17 \approx 0.842$. Note that $\epsilon$-parameterized solutions do not lie on the straight line connecting the Myerson value and the average tree solution. The solution for $\epsilon=0.5$ is not the midpoint of the Myerson value and the average tree solution, either.

Acknowledgements The authors thank anonymous referees for their meticulous reading and valuable comments on the earlier version of this paper. The third author is partly supported by Grant-in-Aid for Scientific Research (C) 22510136. 


\section{References}

1. Berge, C.: Graphs and Hypergraphs. North-Holland, Amsterdam (1973)

2. Borm, P., Owen, G., Tijs, S.: On the position value for communication situations. SIAM Journal on Discrete Mathematics, 5, 305-320 (1992)

3. Chinchuluun, A., Pardalos, P.M., Migdalas, A., Pitsoulis, L.: Pareto Optimality, Game Theory and Equilibria. Springer, Berlin (2008)

4. Demange, G.: On group stability in hierarchies and networks. Journal of Political Economy, 112, 754778 (2004)

5. Herings, P.J.J., van der Laan, G., Talman, A.J.J.: The average tree solution for cycle-free graph games. Games and Economic Behavior, 62, 77-92 (2008)

6. Herings, P.J.J., van der Laan, G., Talman, A.J.J., Yang, Z.: The average tree solution for cooperative games with communication structure. Games and Economic Behavior, 68, 626-633 (2010)

7. Myerson, R.B.: Graphs and cooperation in games. Mathematics of Operations Research, 2, 225-229 (1977)

8. Myerson, R.B.: Conference structures and fair allocation rules. International Journal of Game Theory, 9 , 169-182 (1980)

9. Shapley, L.S.: A value for $n$-person games. Annals of Mathematical Studies, 28, 307-317 (1953)

10. Slikker, M., van den Nouweland, A.: Social and economic networks in cooperative game theory. Kluwer Academic Publishers, Dordrecht (2001)

11. Slikker, M.: A characterization of the position value. International Journal of Game Theory, 33, 505-514 (2005)

12. Talman, A.J.J., Yamamoto, Y.: Average tree solution and subcore for acyclic graph games. Journal of the Operations Research Society of Japan, 51, 203-212 (2008) 